\title{
CHILDHOOD ARTERIAL STROKE
}

J Harry Baumer

C hildhood stroke affects several hundred children in the UK each year and is in the top 10 causes of childhood death. Many children have another medical condition such as congenital heart disease or sickle cell disease. At least $10 \%$ of people with sickle cell disease will have a stroke during childhood. The physical, emotional, and social effects of stroke result in long lasting consequences both for the affected young person, their family, and society as a whole.

The working party that developed this guideline was initiated by the British Paediatric Neurology Association and worked in collaboration with the Royal College of Physicians Intercollegiate group which has produced stroke guidelines for adults. The paediatric stroke guidelines $^{1}$ address the diagnosis, investigation, initial management, and rehabilitation of acute arterial ischaemic stroke in children beyond the neonatal period. Many aspects of rehabilitation are also relevant to children with other causes of stroke (for example, cerebral venous infarction, neonatal stroke, intracranial haemorrhage). However, the recommendations for acute management underline the importance of distinguishing between ischaemic and other categories of stroke. The scope includes the organisation of services; only clinical practice recommendations are included in this summary.

\section{KEY PRACTICE RECOMMENDATIONS \\ Primary prevention}

- Children with haemoglobin SS or S $\beta^{\circ}$ thalassaemia should be screened yearly from the age of 3 years for internal carotid artery or middle cerebral artery velocity $>200 \mathrm{~cm} / \mathrm{s}$ using appropriately trained personnel and transcranial Doppler ultrasound [grade B]

- Children with sickle cell disease who have internal carotid artery/middle cerebral artery velocity $>200 \mathrm{~cm} / \mathrm{s}$ should be offered long term blood transfusion [grade B]

\section{Initial investigations}

- Cross sectional brain imaging is mandatory in children presenting with clinical stroke [grade C]

- Brain magnetic resonance imaging (MRI) is recommended for the investigation of children presenting with clinical stroke [grade $\mathrm{C}$ ]

- Brain MRI should be undertaken as soon as possible after presentation. If brain MRI will not be available within 48 hours, computerised tomography (CT) is an acceptable initial alternative $\sqrt{ }$

- Brain imaging should be undertaken urgently in children with clinical stroke who have a depressed level of consciousness at presentation or whose clinical status is deteriorating

- Any new neurological symptoms or signs in children with sickle cell disease should be evaluated as potentially being caused by stroke 、

- All children with clinical stroke should have regular assessment of conscious level and vital signs $\sqrt{ }$

- Imaging of the cervical and proximal intracranial arterial vasculature should be performed in all children with arterial ischaemic stroke [grade $\mathrm{C}$ ]

- Imaging of the cervical vasculature to exclude arterial dissection should be undertaken within 48 hours of presentation with arterial ischaemic stroke

- Transthoracic cardiac echocardiography should be undertaken within 48 hours after presentation in all children with arterial ischaemic stroke

- All children with arterial ischaemic stroke should be investigated for an underlying prothrombotic tendency. This should include evaluation for protein $\mathrm{C}$ protein $\mathrm{S}$ deficiency, activated protein $\mathrm{C}$ resistance, increased lipoprotein $\mathrm{Lp}(\mathrm{a})$, increased plasma homocysteine, factor V Leiden, prothrombin G20210A and MTHFR TT677 mutations, and antiphospholipid antibodies [grade $\mathrm{C}$ ]

\section{Acute medical management}

- Temperature should be maintained within normal limits [grade D]

- Oxygen saturation should be maintained within normal limits [grade D] 
- Aspirin ( $5 \mathrm{mg} / \mathrm{kg} / \mathrm{day})$ should be given once there is radiological confirmation of arterial ischaemic stroke, except in patients with evidence of intracranial haemorrhage on imaging and those with sickle cell disease $\sqrt{ }$

- In children with sickle cell disease and arterial ischaemic stroke:

- urgent exchange transfusion should be undertaken to reduce percentage haemoglobin S ( $\mathrm{HbS} \%$ ) to $<30 \%$ and raise haemoglobin to $10-12.5 \mathrm{~g} / \mathrm{dl}(6.4-7.8 \mathrm{mmol} / \mathrm{l})$

- if the patient has had a neurological event in the context of severe anaemia (for example, splenic sequestration or aplastic crisis), or if exchange transfusion is going to be delayed for more than four hours, urgent top-up blood transfusion should be undertaken

- Providing there is no haemorrhage on brain imaging, anticoagulation should be considered in children with:

- confirmed extracranial arterial dissection associated with arterial ischaemic stroke $\sqrt{ }$

- cerebral venous sinus thrombosis [grade C]

\section{Secondary prevention}

- Patients with cerebral arteriopathy other than arterial dissection or moyamoya syndrome or those with sickle cell disease should be treated with aspirin ( $1-3 \mathrm{mg} / \mathrm{kg} /$ day)

- Anticoagulation should be considered:

- until there is evidence of vessel healing, or for a maximum of six months, in patients with arterial dissection

- if there is recurrence of arterial ischaemic stroke despite treatment with aspirin

- until there is evidence of recanalisation or for a maximum of six months after cerebral venous sinus thrombosis $\sqrt{ }$

- In children with sickle cell disease:

- regular blood transfusion (every 3-6 weeks) should be undertaken to maintain the $\mathrm{HbS} \%<30 \%$ and the haemoglobin between $10-12.5 \mathrm{~g} / \mathrm{dl}$ (6.4-7.8 mmol/l) [grade C]

- transfusion may be stopped after two years in patients who experienced stroke in the context of a precipitating illness (for example, aplastic crisis) and whose repeat vascular imaging is normal at this time [grade C]

- after three years a less intensive regime maintaining $\mathrm{HbS} \%<50 \%$ may be sufficient for stroke prevention [grade $\mathrm{C}$ ]

- those who cannot receive regular blood transfusions because of allo-immunisation, auto-antibody formation, lack of vascular access, or non-compliance with transfusion or chelation may be considered for treatment with hydroxyurea [grade $\mathrm{C}$ ]

- Advice should be offered regarding preventable risk factors for arterial disease in adult life, particularly smoking, exercise, and diet [grade D]

- Blood pressure should be measured annually to screen for hypertension 、

\section{Early disability assessment and management}

- As soon as possible after admission, all children following stroke should have an evaluation of:

- swallowing safety [grade D]

- feeding and nutrition

- communication [grade D]

- pain [grade D]

- moving and handling requirements [grade D]
- positioning requirements $\sqrt{ }$

- risk of pressure ulcers [grade D]

- All children affected by stroke should have a multidisciplinary assessment within 72 hours of admission to hospital

- The professionals involved in the acute assessment and management of the child should initiate early liaison with their counterparts in the community to ensure a smooth transition of care $\sqrt{ }$

- Children affected by stroke should be offered advice on, and treatment aimed to achieve, play, self care, leisure, and school related skills that are developmentally relevant and appropriate to their home, community, and school environment [grade D]

- Equipment which is appropriate in meeting rehabilitation aims should be assessed on an individual basis, provided in a timely manner, and regularly monitored by appropriate professionals [grade D]

- Sensorimotor therapies should be practised within a neurological framework, and complement other interventions to improve functional skills $\sqrt{ }$

- Rehabilitation activities should be task orientated and relevant to the individual's life [grade $\mathrm{C}$ ]

- Treatment should be integrated into the child's daily home and school activities [grade D]

- Children should be given as much opportunity as possible to practise skills [grade $\mathrm{C}$ ]

- The assessment tools selected should be appropriate for the child's age, developmental, and functional level $\sqrt{ }$

- Standardised and validated assessment tools should be used where possible $\sqrt{ }$

\section{Motor function}

- Muscle strengthening should be used as part of the therapy programme to prevent or reverse contractures [grade D]

- Muscle strengthening activities should be used to improve functional activity [grade D]

- If spasticity results in functional limitation or discomfort, botulinum toxin injection may be used to reduce muscle tone and improve range of joint motion [grade B]

- The most effective dose for gastrocnemius injection is $20 \mu \mathrm{g} / \mathrm{kg}$ to reduce the dynamic component of muscle shortening and increase active length [grade $\mathrm{C}$ ]

- Botulinum toxin should not be used in isolation from other treatment interventions $\sqrt{ }$

- A rigid ankle foot orthosis (AFO) could be considered to aid standing balance, swing through in gait, and prevention of foot and ankle contractures $\sqrt{ }$

- A hinged or posterior leaf spring AFO should be used to facilitate normal gait patterns [grade D]

- Rehabilitation professionals should consider the presence of somatosensory impairment and integrate this in planning and implementing rehabilitation $\sqrt{ }$

\section{Sensory function and pain}

- Vision and hearing should be assessed as part of the multidisciplinary assessment $\sqrt{ }$

- Children affected by stroke should be assessed for the presence of pain using a validated paediatric pain tool [grade D]

- All pain should be treated actively, using appropriate measures including positioning, handling, and medication $\sqrt{ }$ 
Language and communication

- Professionals working with children affected by stroke should be aware that language and communication skills may be affected [grade D]

- A detailed assessment of the child's communication abilities should be carried out in collaboration with the child, parents/carers, teachers, and other therapists to identify the child's strengths and weaknesses and plan intervention that aims to increase functional abilities. [grade D]

\section{Cognition and emotion}

- Professionals working with children affected by stroke should be aware that cognitive function may be affected, both immediately and in the longer term [grade $\mathrm{C}$ ]

- A detailed psychological assessment of the child's cognitive and functional abilities together with any wider family concerns should be carried out in collaboration with the child, parents/carers, and teachers to identify any special educational needs [grade D]

- Cognitive assessment should take account of the presence of any visual or hearing deficits

- Families and professionals should be aware that stroke may have effects on mood and behaviour [grade D]

- The psychological assessment of the child should include evaluation of mood and behaviour, including wider family concerns [grade D]. This should be undertaken in conjunction with cognitive assessment

- Mood and behaviour should be assessed if there is a change in the child's functioning in the home or school environment $\sqrt{ }$

- Therapists working with a child affected by stroke should assess the child's ability to perform daily living activities $\sqrt{ }$

\section{Transfer to adult services}

- Paediatric general and speciality clinics and Child Development Services should have a local policy on transition to adult services, which should be the responsibility of a named person [grade D]

- A named professional should take responsibility for arranging an introduction to adult health services

- A flexible approach to the timing of this transfer needs to be considered which takes into account the young person's readiness, current health status, and links to other social transitions such as leaving school [grade D]

- A multi-agency transition plan should be formulated for young people with special educational needs, with input from health, education, social services, and the young person to plan transition into further education, training, or employment [grade D]

- A named professional should take responsibility for coordinating the transition plan and ensuring delivery of services [grade D]

\section{Families and carers}

- The medical, social, emotional, and educational needs of the child affected by stroke should be considered early and systematically assessed in a coordinated manner when planning their subsequent care [grade D]

- All members of the health care team should work together with the child and family, using an agreed therapeutic approach [grade D]

- A key worker should be appointed to coordinate the package of care, ensure its delivery, and to act as a central
Recommendation grades and levels of evidence

Grade Evidence

A At least one high quality meta-analysis, systematic review of RCTs, or RCT with a very low risk of bias, and directly applicable to the target population; or a well conducted meta analysis, systematic review of RCTs, or RCT with a low risk of bias or a body of evidence consisting principally of such studies, directly applicable to the target population, and demonstrating overall consistency of results

B A body of evidence including well conducted case-control or cohort studies with a low risk of confounding, bias, or chance and a moderate probability that the relationship is causal, directly applicable to the target population, and demonstrating overall consistency of results; or extrapolated evidence from high quality systematic reviews of case-control or cohort studies, or from high quality case-control or cohort studies with a very low risk of confounding, bias, or chance and a high probability that the relationship is causal

C A body of evidence including well conducted case-control or cohort studies with a low risk of confounding, bias, or chance and a moderate probability that the relationship is causal, directly applicable to the target population and demonstrating overall consistency of results; or extrapolated evidence from high quality systematic reviews of case-control or cohort studies, or from high quality case-control or cohort studies with a very low risk of confounding, bias, or chance and a high probability that the relationship is causal

D Non-analytic studies - for example, case reports, case series, or expert opinion; or extrapolated evidence from well conducted case-control or cohort studies with a low risk of confounding, bias, or chance and a moderate probability that the relationship is causal

No evidence exists; recommended best practice based on the clinical experience of the guideline development group.

$\mathrm{RCT}$, randomised controlled trial.

point of contact for the family [grade D]. The key worker and their role should be explained to the family

- Families/carers should be given factual information about their child's condition as soon as possible after diagnosis [grade D]. This should be simple and consistent, avoiding technical terms and jargon

- Written information should be provided to the child and family regarding the child's health and the statutory and voluntary services available [grade D]

- Children should be given information about their condition at an appropriate level [grade D]

- The child and family should be involved in making decisions about the child's care, including rehabilitation and education [grade D]

\section{Terminology}

- Each team should use a consistent framework and terminology in providing care to children affected by stroke $\sqrt{ }$

- It is recommended that the World Health Organization's International Classification of Functioning (ICF) terminology is used $\sqrt{ }$

\section{COMMENTARY}

The guideline is underpinned by a thorough literature search. Individual recommendations are linked to available references. It is evident that for a large proportion of recommendations there is neither evidence nor existing guidance, indicating the need for further health service research. A list of contacts for useful organisations for children and young people affected by stroke is included.

Membership of the guideline development group included consultant paediatric neurologists, a paediatric 
neuroradiologist, haematologist, and neurosurgeon, and a community paediatrician; an occupational therapist, clinical psychologists, speech and language therapist, physiotherapists, clinical nurse specialists, and patient representatives. The membership did not include a general paediatrician or general practitioner. No formal consensus process was used to agree recommendations where no guidance existed. However, a multi-professional peer group reviewed the guideline. This is important given the lack of available evidence.

Children affected by stroke and their parents were invited to attend a structured workshop in order to identify areas that they thought should be addressed within the guidelines. The key questions arising from this meeting are included in the guideline. Illustrative quotes from children and families are also included.

In summary, this guideline provides comprehensive evidence based recommendations on acute stroke in children beyond the neonatal period. It strongly recommends regular Doppler ultrasound screening in children with sickle cell disease, with regular transfusion for those at high risk, to prevent stroke.

\section{REFERENCE}

1 Intercollegiate Working Party for Paediatric Stroke. Clinical guidelines for diagnosis and management of acute stroke in childhood. London: Royal College of Physicians, anticipated publication date 2004.

\section{ARCHIVIST}

\section{Heart abnormalities associated with ventricular septal defect}

B etween two and six of every thousand babies born alive have a ventricular septal defect (VSD) and overall just under $90 \%$ survive to young adult life. Other cardiac malformations such as infundibular pulmonary stenosis, persistent ductus arteriosus, and coarctation of the aorta may be associated with VSD but there has been no large prospective series of patients studied with modern ultrasound technology including Doppler colour flow mapping. Now such a series has been reported from Stirling, Scotland (S Glen and colleagues. Heart 2004; ht 25007)

The study included 1448 patients with congenital VSD identified between 1991 and 1998 most of them at a paediatric cardiology centre and some (probably about a third) from a congenital heart disease clinic for adults. Patients with conotruncal abnormalities (transposition of the great arteries, tetralogy of Fallot, persistent truncus arteriosus or double outlet right ventricle), univentricular heart, or atrioventricular septal defects, were excluded. Overall, the most common associated cardiac abnormalities were infundibular pulmonary stenosis $(5.8 \%)$, aortic prolapse $(3.6 \%)$, pulmonary valvar stenosis $(2.7 \%)$, ostium secundum atrial septal defect $(2.2 \%)$, persistent ductus arteriosus $(1.9 \%)$, and coarctation of the aorta ( $1.5 \%$ ). Because the adult group had often had previous surgery or percutaneous intervention certain abnormalities were less prevalent in the adult than the paediatric group (ostium secundum ASD 1.5\% vs 2.8\%, persistent ductus arteriosus $0.5 \%$ vs $2.9 \%$, coarctation of the aorta $0.3 \%$ vs $2.4 \%)$. Aortic valve prolapse (6.7\% vs $1.3 \%$ ) and infundibular pulmonary stenosis $(7.8 \%$ vs $4.3 \%)$ were more prevalent in the adult group. The VSD was the only cardiac abnormality in $78 \%$ of patients, $20 \%$ had one associated abnormality and $2.5 \%$ two or three. Muscular defects were less prevalent among adult patients (11\% vs 23\%) as a result of spontaneous closure. Forty-four children had chromosomal abnormalities: trisomy 21 (29), trisomy 18 (7), trisomy 13 (4), and 22q11 deletion (4). Seven hundred and forty-three patients were assessed in infancy and $29(4 \%)$ of these died, 28 in infancy. Of the 28, 11 had chromosomal abnormalities, five had other congenital malformation syndromes, two were preterm, and one had immotile cilia. The prevalence of aortic regurgitation increased with age, it being most commonly diagnosed at between 11 and 15 years of age. One hundred and six children (12.6\%) had corrective surgery (including percutaneous closure) at a mean age of 1.4 years. No patient developed endocarditis.

Additional cardiac abnormalities were present in $22 \%$ of these patients with VSD and such abnormalities should be looked for on initial assessment. The authors of this paper recommend follow up to at least the age of 30 years in order to detect secondary aortic regurgitation or infundibular aortic stenosis. 